\title{
Electrosynthesis of Conducting Polymers in Lecithin Liquid Crystal Reaction Field
}

\author{
Kohei Yamabe, Hiromasa Goto \\ Department of Materials Science, Faculty of Pure and Applied Sciences, \\ University of Tsukuba, Tsukuba, Ibaraki 305-8573,Japan \\ Correspondence to: H. Goto (E -mail: gotoh@ims.tsukuba.ac.jp) \\ Highlights \\ - Lecithin-formed LC structure in water solution \\ - Preparation of conducting polymer thin films on ITO glass \\ - Lecithin LC structure transcribed to polymer thin films \\ - Island pattern observed in polymer thin films by the SEM.
}

\begin{abstract}
Lecithin is a biological material that exhibits lyotropic liquid crystal form at certain concentrations in water. Specifically, lecithin forms lamellar structures like a smectic phase layers. We describe electro-oxidative preparation of polymers such as polyaniline (PANI), poly(ethylenedioxythiophene) (PEDOT), and polypyrrole (PPy) in a lecithin liquid crystal matrix. Cross-shaped images (Maltese crosses) derived from lamellar structures were observed for the resultant polymer film with a polarizing optical microscopy under crossed nicol condition. Lecithin lamellar structure in the water was transcribed to the resultant polymers during the electropolymerization. This research demonstrates transcription of a liquid crystal aggregation form of biomaterials onto synthetic polymers through electrochemical process.
\end{abstract}

Keywords: lamellar; liquid crystal; polymer; electropolymerization

\section{Introduction}

Conducting polymers, such as polyaniline (PANI), polypyrrole (PPy), and poly(3,4-ethylenedioxythiophene) (PEDOT) are studied as organic semiconductors. Orientation of organic semiconducting polymers is a significant issue for applications.

Electrochemical method is a widely used technique to obtain electrochromic materials. For production of functional polymer film, application of electrolyte solution in electrochemical polymerization has been carried out such as ionic liquid or liquid crystal [1,2]. Previously, a new technique for preparing chiral conducting polymer films in a thermotropic chiral liquid crystal (LC) has been demonstrated [3]. LC magneto-electrochemical polymerization can produce oriented conducting polymers through influence of the liquid crystal orientation matrix under magnetic field [4]. Macroscopic aggregation pattern of the LC was transcribed to the resultant polymer, producing conducting polymers having liquid crystal-like arrangement.

Lecithin is a material of cell surface membranes that forms lipid bilayers in water. This arrangement corresponds to lyotropic LC (LC state in solvent) similar to smectic A phase [5]. The cross-shaped figure referred to as a Maltese cross was observed in smectic A and nematic phase [6].

Many biological molecules show lyotropic LC structure related to maintenance of mechanical form and biological reactions. In this study we carry out electrochemical 
preparation of conducting polymers in lecithin as a cell membrane material to obtain thin organic films showing biological LC-like structure through structure transcription from the LC aggregation form. Biological LC reaction fields may accelerate reaction in a biological system and change conformation through transcription from the molecular level to the macromolecular level. This research may provide a key reaction mechanism to form new biological molecules and tissues in living organisms, and lead to construction of artificial tissues by synthetic polymers.

\section{Materials}

\section{Experimental}

Aniline was purchased from Wako Chemical (Japan), and purified by distillation. Lecithin, pyrrole, EDOT and tetrabutylammonium perchlorate were purchased from Tokyo Chemical Industry (TCI, Japan), and used as received.

\section{Instruments}

Fourier Transform Infrared absorption was measured with a FT-IR 4600 (Jasco) by using the $\mathrm{KBr}$ method. UV-vis optical absorption spectroscopy measurements were carried out with a V-630 UV-vis optical absorption spectrometer (Jasco, Japan). Optical textures were observed by polarizing optical microscopy (POM) using an ECLIPS LV 100 polarizing microscope (Nikon, Japan). Polymer surfaces were observed by scanning electron microscopy (SEM) using a JSM-7000F (JEOL, Japan). Cyclic voltammetory were carried out with a $\mu$ AUTOLAB TYPE III (ECO Chemie).

\section{Preparation of polymer films}

We used Lecithin $50 \mathrm{wt} \%$ water solution which exhibits LC phase at room temperature $[7,8]$. First, lecithin and monomer were added to distilled water. Table 1 shows constituents and quantity of the compounds for the present electrochemical polymerizations. The LC electrolyte solution containing monomer was injected between two indium-tin-oxide-coated (ITO) glass slides sandwiched with a Teflon sheet as spacer. A constant voltage of $12.0 \mathrm{~V}$ was applied for 20 minutes at room temperature to carry out electrochemical oxidative polymerization at the anode side of the ITO, which we refer to as sandwich cell polymerization. Polymer thin films on the ITO glass were washed with hexane and THF to obtain pure polymer films.

Table 1. Electrochemical polymerization in lecithin liquid crystal.

\begin{tabular}{lcccl}
\hline & $\begin{array}{c}\text { Monomer } \\
\text { Quantity }(\mathrm{mg})\end{array}$ & $\begin{array}{c}\text { Lecithin } \\
(\mathrm{g})\end{array}$ & $\mathrm{H}_{2} \mathrm{O}(\mathrm{g})$ & \multicolumn{1}{c}{ Film $^{1}$} \\
\hline Aniline & 23.0 & 1.41 & 1.40 & PANI $^{2}$ \\
Py $^{3}$ & 15.2 & 0.54 & 0.50 & PPy $^{4}$ \\
EDOT $^{5}$ & 20.3 & 0.65 & 0.60 & PEDOT $^{6}$ \\
\hline
\end{tabular}

\footnotetext{
${ }^{\mathrm{I}}$ Resultant polymer film.

${ }^{2}$ Polyaniline.

${ }^{3}$ Pyrrole.

${ }^{4}$ Polypyrrole.

53,4-ethylenedioxythiophene

${ }^{6}$ Poly(3,4-ethylenedioxythiophene).
} 


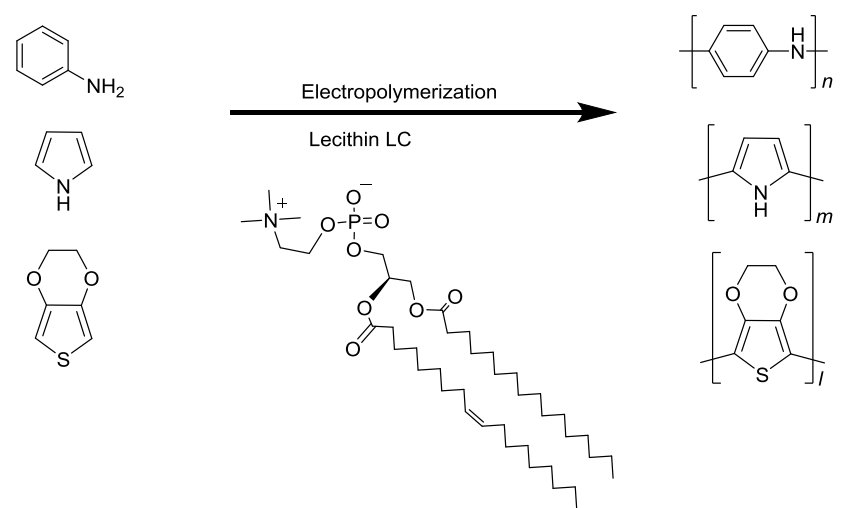

Scheme 1. Electrochemical polymerization in lecithin liquid crystal.

\subsection{Characterization}

Figure 1 shows Fourier transform infrared (FT-IR) spectra of PANI, PPy, and PEDOT synthesized with electropolymerization. The resultant polymers was peeled off from ITO glass and the powder sample were measured using the pressed $\mathrm{KBr}$ disk method. The polymers shows no characteristic absorption band derived from lecithin such as $1740 \mathrm{~cm}^{-1}$ and $2800-2950 \mathrm{~cm}^{-1}$. This result indicated that lecithin LC was removed by washing from polymer films.

IR absorption of the PANI: FTIR (KBr, $\left.\mathrm{cm}^{-1}\right)$ : 1060, $1105(\mathrm{C}-\mathrm{H}$ in-plane. on 1,4-ring and 1,2,4-ring); 1240 (B-B-B str.); 1315 (B-B-Q str.); 1510 (N-B-N str.); 1587 (N=Q=N str.); $3380\left(\mathrm{NH}_{2}\right.$ sym. str., $\mathrm{NH}$ str.) [9].

IR absorption of the PPy are as follows: FTIR $\left(\mathrm{KBr}, \mathrm{cm}^{-1}\right): 1022,1090(\mathrm{C}-\mathrm{H}$ in-plane); 1261 (C-N str.); 1542, 1384 (C-H fundamental vibration in pyrrole ring) [10].

IR absorption of the PEDOT are as follows: FTIR $\left(\mathrm{KBr}, \mathrm{cm}^{-1}\right): 691,842,978$ (vibration modes of $\mathrm{C}-\mathrm{S}$ bond in thiophene ring); 1093, 1213 (str. ethylenedioxy group); 1339, 1483, 1518 ( $\mathrm{C}=\mathrm{C}$ and $\mathrm{C}-\mathrm{C}$ str. in thiophene ring) [11].

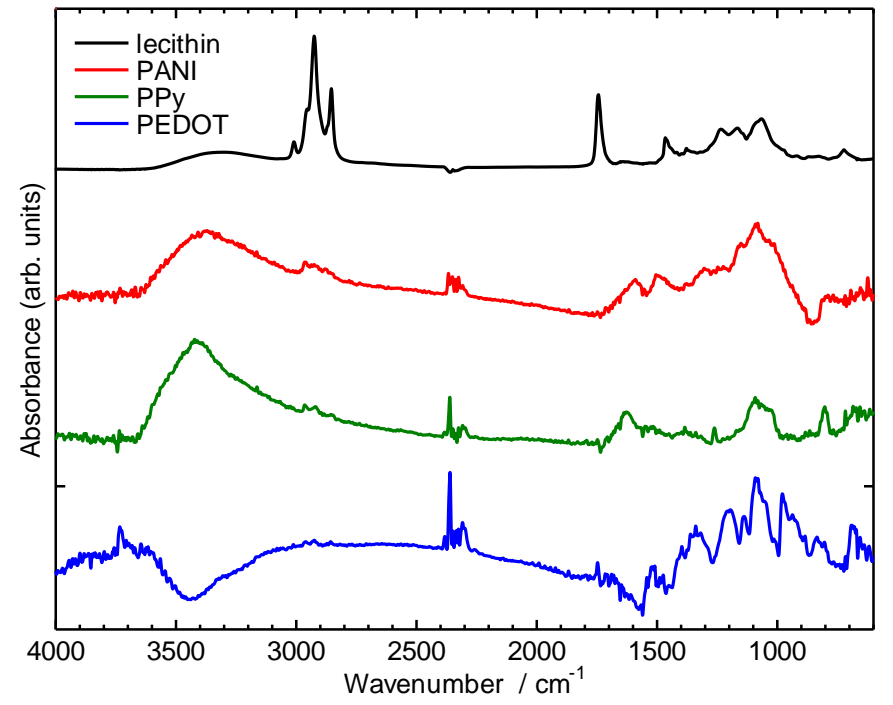

Figure 1. FT-IR absorption spectra of lecithin, polyaniline (PANI), polypyrrole (PPy), and Poly(3,4-ethylenedioxythiophene) (PEDOT) prepared in lecithin liquid crystal. 
K. Yamabe, H. Goto,

Electrosynthesis in Lecithin Liquid Crystal

Fibers and Polymers 2018, Vol.19, No.1, 248-253

\section{Result and discussion}

\section{UV-vis}

UV-vis absorption spectra of the polymer thin films obtained in lecithin LC (PANI, PPy and PEDOT) are shown in Figure 2. PANI shows absorption band at $427 \mathrm{~nm}$ due to $\pi-\pi^{*}$ transition of the main chain. PPy and PEDOT show absorption band of $\pi-\pi^{*}$ transition 444 and $449 \mathrm{~nm}$ respectively. The absorption bands at 400-500 $\mathrm{nm}$ are derived from $\pi-\pi^{*}$ transition of the conjugated main chain. This absorption band indicated $\pi$-conjugated system of the polymer.

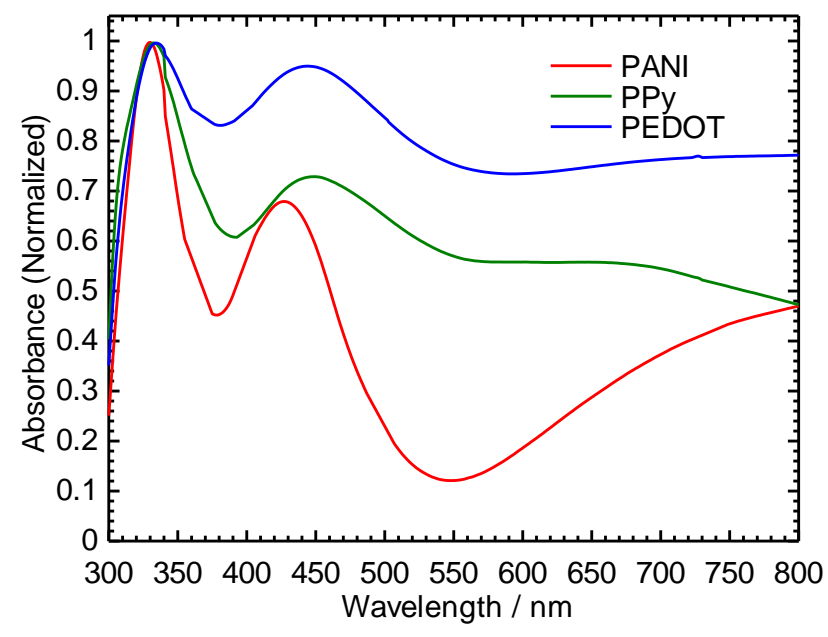

Figure 2. UV-vis absorption spectra of polyaniline (PANI), polypyrrole (PPy), and Poly(3,4-ethylenedioxythiophene) (PEDOT) prepared in lecithin liquid crystal.

\section{POM}

Surface structure of lecithin in the LC state and the resultant polymer films were observed with the POM. The POM image of lecithin in the water is shown in Figure 3. This image showing unique fingerprint pattern visually confirms that lecithin forms LC in water. Cross-shaped figures derived from spherical structure of lecithin LC are partly found in Figure 3 (right) as a form of Maltese cross. The POM images of the resultant polymer films are shown in Figure 4. PANI displays quite similar texture to that of lecithin LC, Figure 3 (left). Some Maltese crosses are also seen for the PEDOT film prepared in the lecithin LC electrolyte solution, Figure 5. These Maltese crosses are due to transcription of LC structure from the lecithin LC during the polymerization. Figure 6 shows a POM image with insertion of a gypsum red color plate in the optical path, additionally. Maltese crosses can appear blue or yellow along the optical axis of the sample under the observation of the POM with the gypsum plate $[6,12,13]$. The top right and bottom left areas appear blue and the top left and bottom right areas appear yellow in the left image, Figure 6 . Rotation of a $\lambda$-plate during the observation changes the color. The top right and the bottom left areas appear yellow, and the top left and the bottom right areas appear blue in the right image with polarizer rotation of the POM at $45^{\circ}$. The change in color indicates that the polymer film forms crystal-like structure (derived from LC-like structure), although the polymers are amorphous in general, Figure 6 [6]. 
K. Yamabe, H. Goto,

Electrosynthesis in Lecithin Liquid Crystal

Fibers and Polymers 2018, Vol.19, No.1, 248-253

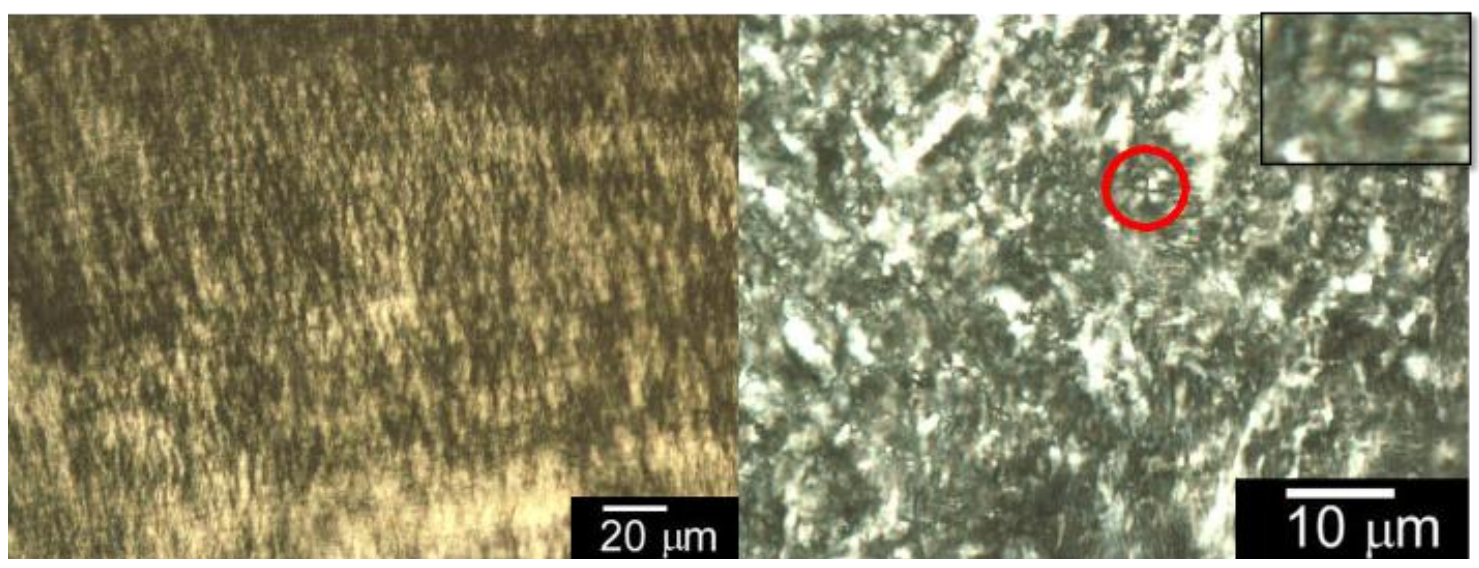

Figure 3. Polarizing optical microscopy (POM) images of lecithin LC, (inset) crossshaped figures of lecithin liquid crystal (LC) lamellar structure.

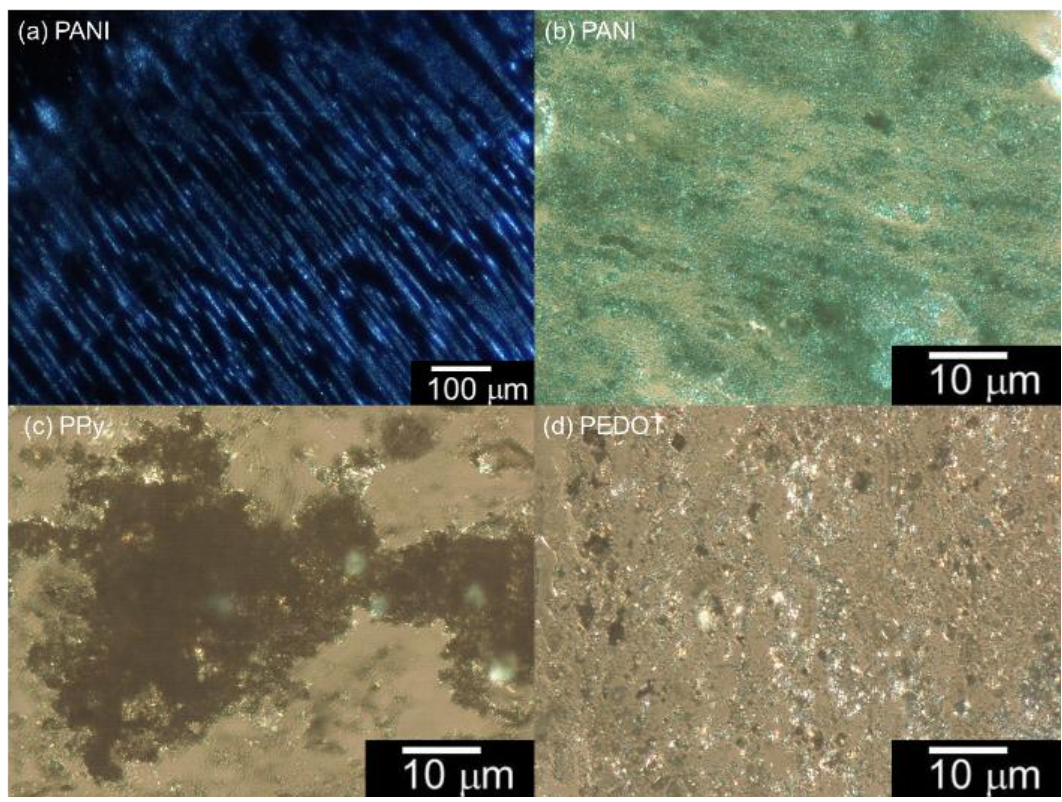

Figure 4. POM images of (a,b) PANI; (c) PPy; (d) PEDOT.

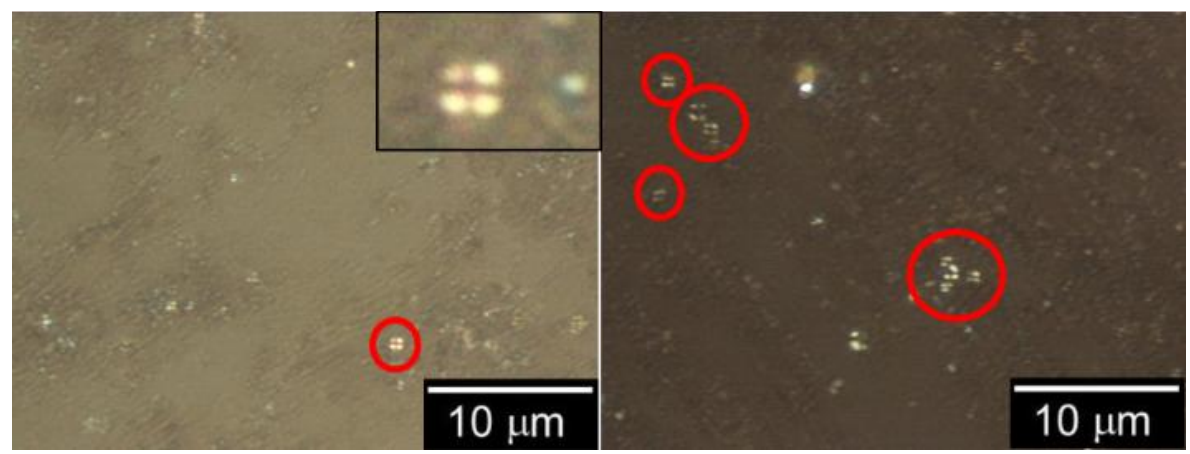

Figure 5. POM images of PEDOT with insertion of gypsum red color plate. 


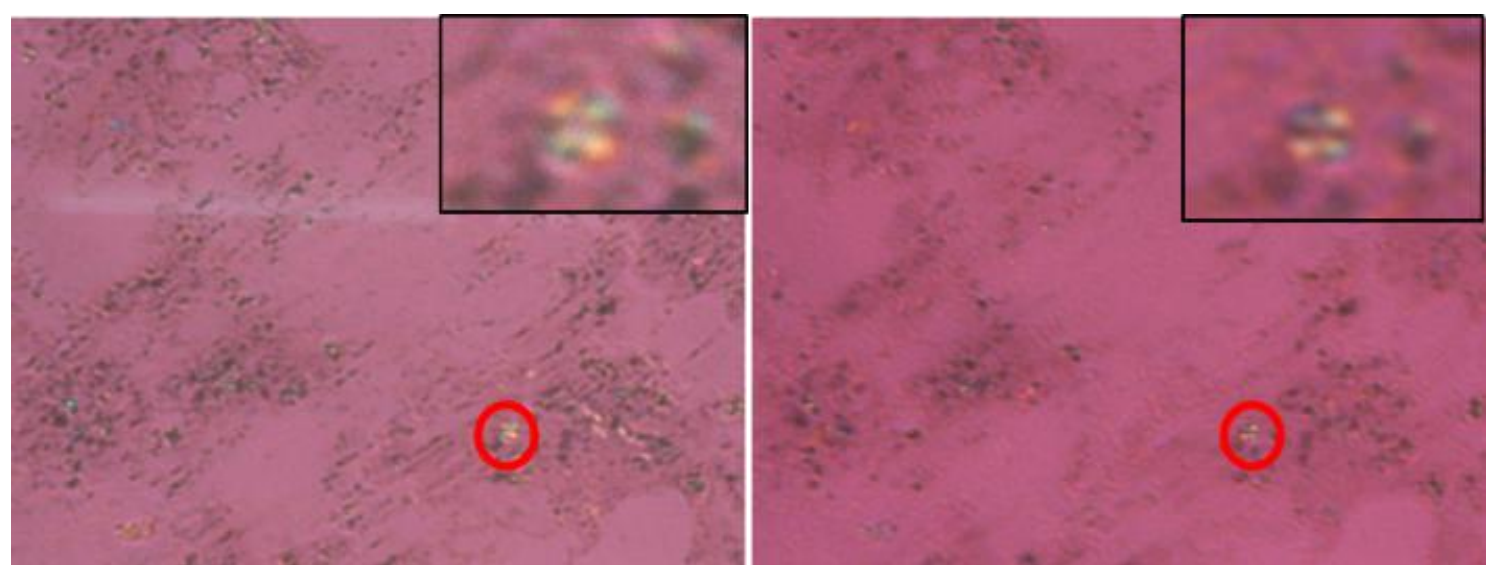

SEM

Figure 6. POM images of PEDOT with cross figures.

Surface structure of the polymer films on the ITO glass (substrate) was observed with the SEM for investigation of polymer fine structure in consideration with the lecithin LC as a molecular template. The SEM images of the polymer films are shown in Figure 7. The PANI thus synthesized in this study displays short fibril structure, Figure 7 (a). Fibril structure is often observed on the surfaced of PANI obtained with electrosynthesis $[15,16]$. The PPy shows pebble-like structure. Previous study evaluated that PPy synthesized with electro-polymerization can form sphere morphology [17]. The PEDOT surface shows island-like structure. Electrosynthesis of PEDOT in isotropic solvent such as acetonitrile and propylene carbonate shows unique structures [18]. However, this island-like structure obtained in this research has not been reported to date, indicating lecithin LC functions for production of this new morphology.

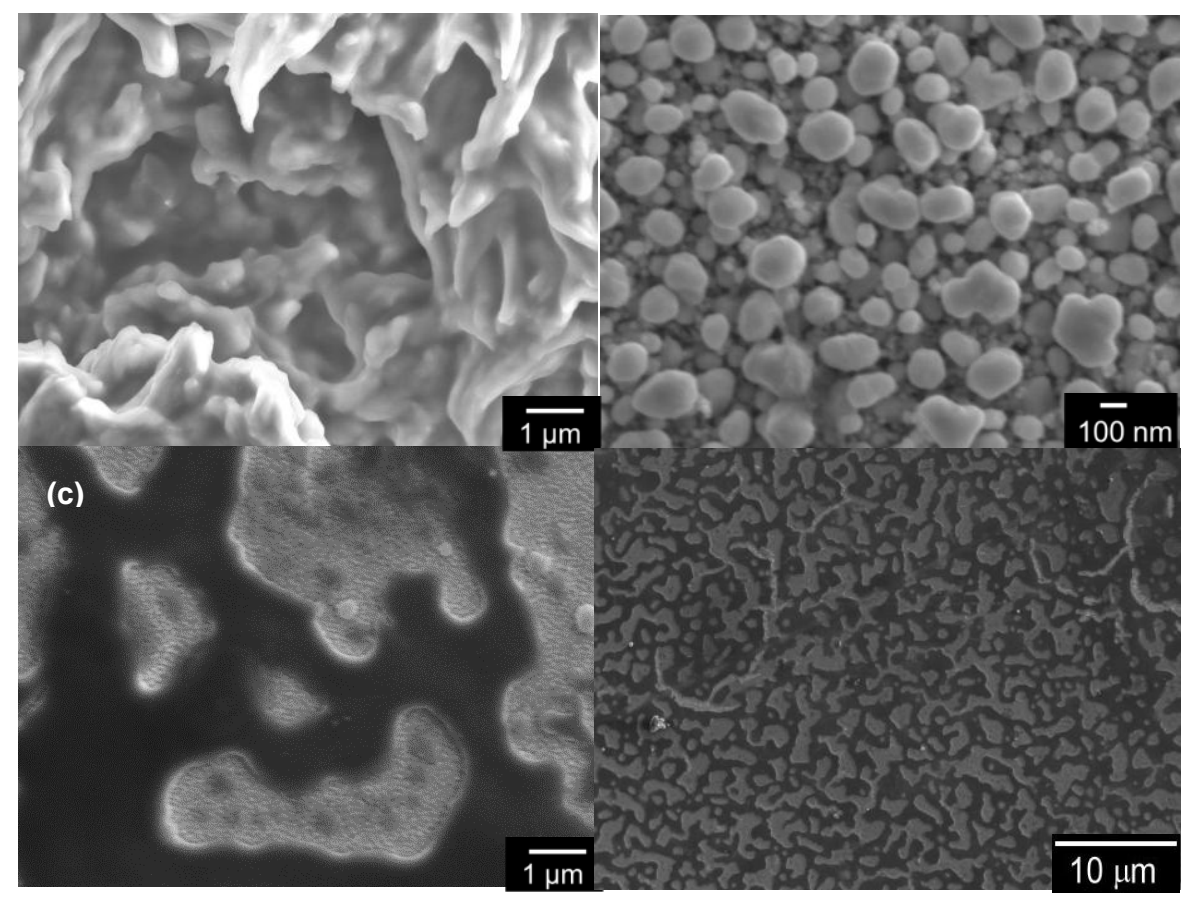

Figure 7. SEM image of (a) PANI; (b) PPy; (c) (d) PEDOT. 
K. Yamabe, H. Goto,

Electrosynthesis in Lecithin Liquid Crystal

Fibers and Polymers 2018, Vol.19, No.1, 248-253

CV

Cyclic voltammetry (CV) analysis of the PANI, the PPy and the PEDOT (prepared with lecithin LC electrolyte solution) were carried out at various scan rates of 20, 40, 60, 80 and $100 \mathrm{mV} / \mathrm{s}$. The CV cell contains a polymer film on ITO glass as a working electrode, a platinum wire counter electrode, and an $\mathrm{Ag} / \mathrm{AgNO}_{3}$ reference electrode. Electrolyte solution contained $0.1 \mathrm{M}$ tetrabutylammonium perchlorate in acetonitrile. $\mathrm{CV}$ analysis reveals electrochemical property of the polymer films such as oxidation-reduction potential. The CV results were shown in Figure 8. A redox signal was observed in all polymer films. Oxidation curve indicates occurrence of electrochemical doping. Reduction curve indicates electrochemical de-doping [19-21]. The current value of the redox signals gradually increased with the increase of scan rates. These results reveal repeatable oxidation-reduction process as doping and de-doping.
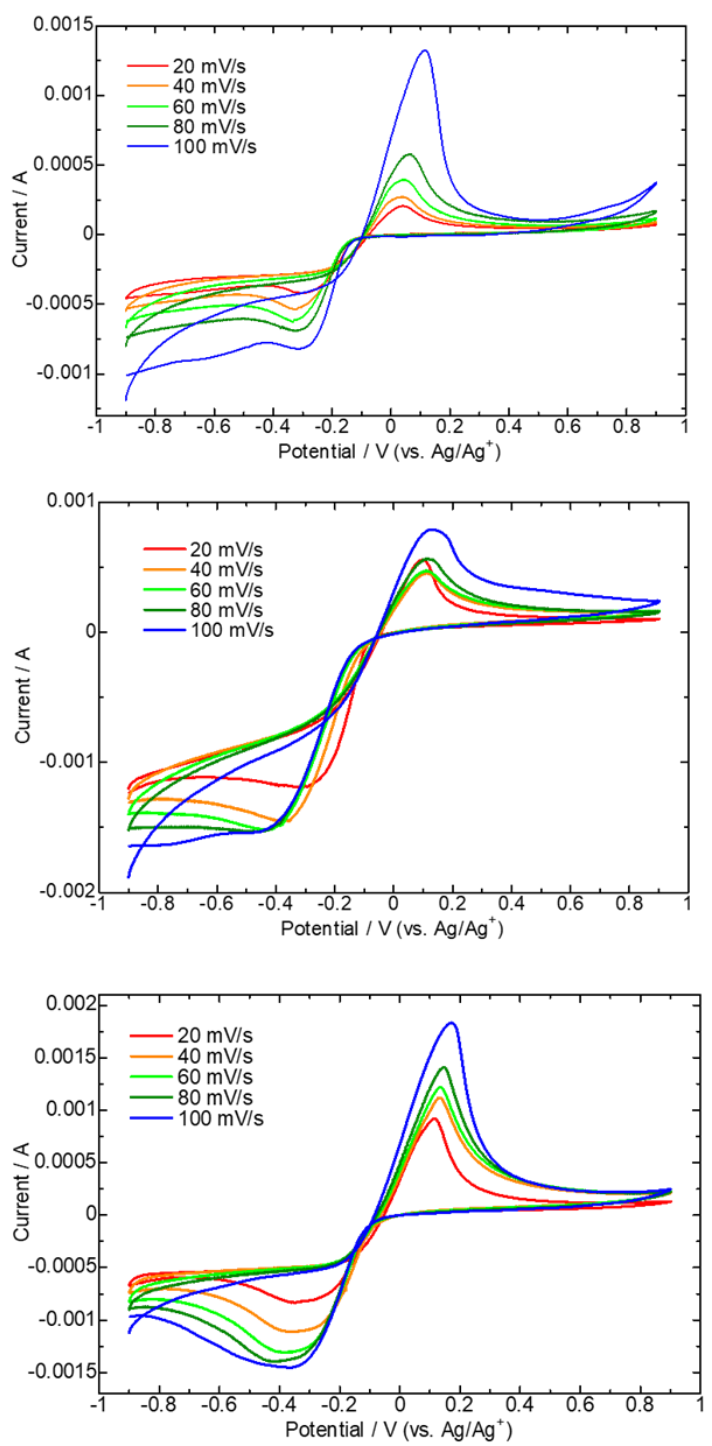

Figure 8. Cyclic voltammogram of (a) PANI; (b) PPy; (c) PEDOT in $0.1 \mathrm{M} / \mathrm{TBAP}$ acetonitrile solution. 
K. Yamabe, H. Goto,

Electrosynthesis in Lecithin Liquid Crystal

Fibers and Polymers 2018, Vol.19, No.1, 248-253

\section{Conclusion}

Preparation of conducting polymers showing unique structure was carried out in lecithin LC. Polymer film morphology, aggregation and orientation are important for applications of electro-device. The unique texture comes from transcription of the LC structure of lecithin in the water during the polymerization. Many biomaterials form LC in water, such as cells, muscle, brain, etc. Thus, the LC structure in biological systems may be integrated to change the conformation and biological reactions in living organisms. This research is an example of molecular transcription electrochemical reactions from self-aggregation of biomolecules and an approach for production of polymers having biological tissue-like structure.

\section{Acknowledgements}

This work was supported by Grants-in-Aid for Scientific Research, Japan (KAKENHI, No. 17K05985) and NIMS microstructural characterization platform as a program of "Nanotechnology Platform" of the Ministry of Education, Culture, Sports, Science and Technology (MEXT), Japan. We would like to thank the OPEN FACILITY, Research Facility Center for Science and Technology, University of Tsukuba, for supporting measurement.

\section{REFERENCE}

1. Lu, B., Zhang, S., Qin, L., Chen, S., Zhen, S., and Xu, J. Electrochimica Acta, 106, 201-208 (2013).

2. Lu, B., Yan, J., Xu, J., Zhou, S., and Hu, X. Macromolecules, 43, 4599-4608 (2010).

3. H. Goto, and K. Akagi. Macromol. Rap. Commun., 25, 1482-1486 (2004).

4. Kawabata, Kohsuke, Shigeki Nimori, and Hiromasa Goto. ACS Macro Lett. 2.7, 587-591 (2013).

5. The Chemical Society of Japan, "Chemistry of Liquid Crystal”, Gakkaishuppan, Tokyo, 1994.

6. I. Dirking, "Textures of Liquid Crystals", Wiley VCH, Manchester, 2003.

7. D. Chapman, R.M. Williams, and B.D. Ladbrooke, Chem. Phys. Lip., 1, 445-475 (1967).

8. G. Rong, J. Yang, S.E. Friberg, P.A. Aikens, and J. N. Greenshields, Langmuir 12, 4286-4291 (1996).

9. Tang, J., Jing, X., Wang, B., and Wang, F., Synth. Met., 24.3, 231-238 (1988).

10. He, C., Yang, C., and Li, Y., Synth. Met., 139.2, 539-545 (2003).

11. Selvaganesh, S. V., Mathiyarasu, J., Phani, K. L. N., and Yegnaraman, V., Nanoscale Research Lett., 2.11, 546 (2007).

12. E. Brasselet, N. Murazawa, H. Misawa, and S. Juodkazis, Phys. Rev. Lett., 103, 103903 (2009).

13. M. Harms, S. Mackeben, and C.C. Müller-Goymann, Physicochem. Eng. Asp., 259, 81-87 (2005).

14. D.T. Fluck, A.F. Henson, and D. Chapman. J. Ultrastruct. Res., 29, 416-429 (1969).

15. Tao, Y., Li, J., Xie, A., Li, S., Chen, P., Ni, L., and Shen, Y., Chem. Commun., 50.84, 12757-12760 (2014).

16. Ye, J. R., Zhai, S., Gu, Z. J., Wang, N., Wang, H., and Shen, Q., Mater. Lett., 132, 377-379 (2014). 
K. Yamabe, H. Goto,

Electrosynthesis in Lecithin Liquid Crystal

Fibers and Polymers 2018, Vol.19, No.1, 248-253

17. Navale, S. T., Mane, A. T., Chougule, M. A., Sakhare, R. D., Nalage, S. R., and Patil, V. B., Synth. Met., 189, 94-99 (2014).

18. Poverenov, E., Li, M., Bitler, A., and Bendikov, M., Chem. Mater., 22.13, 4019-4025 (2010).

19. Diaz, A. F., Castillo, J. I., Logan, J. A., and Lee, W. Y., J. Electroanal. Chem. Interfacial Electrochem., 129, 115-132 (1981).

20. Yan, B., Yang, J., Li, Y., and Qian, R., Synth. Met., 58, 17-27 (1993).

21. Genies, E. M., and Lapkowski, M. S., J. Electroanal. Chem. Interfacial Electrochem., 220, 67-82 (1987). 